and Ermalov ${ }^{7}$ who concluded from studies of the phosphorescence spectra of mixtures of benzophenone (and other donors) and naphthalene in ether plus ethanol at $77^{\circ} \mathrm{K}$ that there was a transfer of energy between the triplet states of these molecules in the rigid glass solutions.

Our results also suggest a possible explanation of the observations of Ferguson and Tinson ${ }^{8}$ who found that the phosphorescence spectrum of benozphenone in petroleum ether at $-180^{\circ} \mathrm{C}$ is replaced by another at longer wavelengths when the concentration is increased from $10^{-4}$ to $10^{-2} M$. These workers attributed their results to energy transfer to the triplet state of benzophenone. As we have shown that the triplet state of this molecule reacts very rapidly at $77^{\circ} \mathrm{K}$ with the components of E.P.A. to form a ketyl radical, it is possible that the second phosphorescence spectrum observed by Ferguson and Tinson comes from the ketyl radical or some resulting compound.

We wish to thank the National Research Council of Canada for generous grants in support of this research.

\footnotetext{
${ }^{1}$ C. A. Hutchison, Jr., and B. W. Mangum, J. Chem. Phys. $29,952(1958) ; 34,908$ (1961)

2 J. H. van der Waals and M. S. de Groot, Mol. Phys. 2, 233 (1959).

${ }^{3}$ M. S. de Groot and J. H. van der Waals, Mol. Phys. 3, 190 (1960).

4 E. Clar, Spectrochim. Acta 4, 116 (1950).

5 E. H. Gilmore, G. E. Gibson, and D. S. McClure, J. Chem. Phys. 20, 829 (1950); 23, 399 (1955).

6 D. H. Henderson and C. A. McDowell (unpublished).

${ }^{7}$ A. Terenin and V. Ermalov, Doklady Akad. Nauk S.S.S.R. 85, 547 (1952); Trans. Faraday Soc. 52, 1042 (1956).

${ }^{8} \mathrm{~J}$. Ferguson and H. J. Tinson, J. Chem. Soc. 1952, 3083.
}

\section{Sixth Virial Coefficients for Gases of Parallel Hard Lines, Hard Squares, and Hard Cubes}

William G. Hoover* and ANDrew G. De Rocco

Department of Chemistry, University of Michigan, Ann Arbor, Michigan

(Received January 9, 1961)

$\mathbf{T}$

VHE virial equation of state can be written as

$$
\beta p=\sum_{1}^{\infty} B_{n} \rho^{n}
$$

where $\beta=1 / k T, \rho$ is the number density, and the set of coefficients $\left\{B_{n}\right\}$ are the virial coefficients. The topological graphs are known which permit the calculation of $\left\{B_{n}\right\}$ up to $B_{7},{ }^{1}$ if the related integrals of the $f_{i j}$ products can be accomplished. Because the integrals are formidable for realistic choices of the potential, simpler models have been introduced to effect the integrals. The simplest nontrivial case is that of parallel hard lines, squares, and cubes. ${ }^{2}$ The hard-cube potential has the property that any integral $I_{n}$ of a product $\pi f_{i j}$ can be written as $I_{1}\left|I_{1}\right|^{n-1}$, where $n$ is the dimensionality of the interacting figures; this feature of the problem was clearly noted by Zwanzig. ${ }^{3}$ To compute the set $\left\{B_{n}\right\}$ one need only evaluate the pertinent set of integrals in one dimension; the extension to higher dimensions involves only mechanical effort. To calculate $B_{2}$ and $B_{3}$, only one integral must be done. For $B_{4}, B_{5}$, and $B_{6}$ one needs to accomplish three, 10, and 56 integrals, respectively.

We have succeeded in computing $B_{6}$ by a straightforward but tedious method, the details of which will be published. We present in Table I the results for $B_{6}$

TABLE I. Virial coefficients for lines, squares, and cubes.

\begin{tabular}{llll}
\hline & Lines & Squares & Cubes \\
\hline$B_{1}$ & 1 & 1 & 1 \\
$B_{2}$ & 1 & 2 & 4 \\
$B_{3}$ & 1 & 3 & 9 \\
$B_{4}$ & 1 & $11 / 3$ & $34 / 3$ \\
$B_{5}$ & 1 & $67 / 18$ & $455 / 144$ \\
$B_{6}$ & 1 & $121 / 40$ & $-2039 / 108$ \\
$B_{1}$ & 1.0000 & 1.0000 & 1.0000 \\
$B_{2}$ & 1.0000 & 1.0000 & 1.0000 \\
$B_{3}$ & 1.0000 & 0.7500 & 0.5625 \\
$B_{4}$ & 1.0000 & 0.4583 & 0.1771 \\
$B_{5}$ & 1.0000 & 0.2326 & 0.0123 \\
$B_{6}$ & 1.0000 & 0.0945 & -0.0184 \\
\hline
\end{tabular}

and the lower coefficients (previously known), for lines of length $\sigma$, squares of area $\sigma^{2}$, and cubes of volume $\sigma^{3}$. The first set of values corresponds to the choice of $\sigma^{n}$ for the $n$-dimensional $B_{2}$; the second set uses $2^{n-1} \sigma^{n}$ for the corresponding $B_{2}$ (both sets are employed in the literature). It is interesting to note that $B_{6}$ is negative in three dimensions, a result anticipated by Temperley on the basis of his extension of the Zwanzig calculations to higher dimensions. ${ }^{4}$ At this point the earlier work deserves some further comment:

(1) Tonks $s^{5}$ calculated the equation of state for the one-dimensional case, but the fact that Tonks did not employ the cluster expansion prevents any extension of his results to higher dimensions.

(2) Geilikman ${ }^{2}$ obtained $B_{2}, B_{3}$, and $B_{4}$ for cubes, although, as he later pointed out, the result for $B_{4}$ was incorrect.

(3) Zwanzig, ${ }^{3}$ using the results of Riddell and Uhlenbeck, ${ }^{6}$ computed the first five coefficients for the case described in this letter. Zwanzig agrees that the inequalities in Helmholtz energy for systems of cubes and spheres used in his paper ${ }^{3}$ to limit the virial coefficients for spheres do not seem sufficient for this purpose. ${ }^{7}$ If his treatment had been rigorous our result would imply a negative $B_{6}$ for hard spheres, in contrast to the suggestion of Alder and Wainwright ${ }^{8}$ that both $B_{6}$ 
and $B_{7}$ for hard spheres are positive. Whether or not $B_{n}{ }^{\circ}$ and $B_{n}{ }^{\square}$ have the same sign is still an open question.

(4) As noted earlier Temperley found that $B_{4}$ was negative in five dimensions and $B_{5}$ negative in four dimensions. It is clear that $B_{5}$ will eventually become positive again if a sufficient number of dimensions is employed. His later statement ${ }^{9}$ that Ford found $B_{4}$ and $B_{7}$ as negative for spherical molecules is without foundation, since the Gaussian model employed by Ford does not correspond to any potential.

(5) Domb $b^{10}$ presented equations of state for "squares" and "cubes" in which $B_{4}, B_{5}$, and $B_{6}$ were negative. These were presumably approximations derived from an Ising model, but none of the coefficients beyond the first agree with the exact results for hard squares and hard cubes.

We will present the detailed calculation of $B_{6}$ in the near future. We are now actively concerned with the calculation of $B_{7}$ and the analog of the radial distribution function for such systems.

* Proctor and Gamble Predoctoral Fellow, 1960-1961.

${ }^{1} \mathrm{G}$. W. Ford, unpublished dissertation, University of Michigan, Ann Arbor, Michigan, 1954; $n=7$.

2 B. T. Geilikman, Proc. Acad. Sci. U.S.S.R. 70, 25 (1950).

${ }^{3}$ R. W. Zwanzig, J. Chem. Phys. 24, 855 (1956).

${ }^{4}$ H. N. V. Temperley, Proc. Phys. Soc. (London) 70B, 536 (1957).

' L. Tonks, Phys. Rev. 50, 955 (1936).

${ }^{B}$ R. J. Riddell, Jr. and G. E. Uhlenbeck, J. Chem. Phys. 21, 2056 (1953).

${ }^{7}$ R. W. Zwanzig (private conversation).

${ }^{8}$ B. J. Alder and T. E. Wainwright, J. Chem. Phys. 33, 1439 (1960)

${ }^{9}$ H. N. V. Temperley, Nuovo cimento Suppl. 9, Ser. 10, 163 (1958).

${ }^{10}$ C. Domb, Nuovo cimento Suppl. 9, Ser. 10, 9 (1958).

\section{Infrared Spectrum of Diimide*}

Edmund J. Blau, Bernard F. HochHeImer, and HILBERT J. UNGER

Applied Physics Laboratory, The Johns Hopkins University, Silver Spring, Maryland

(Received December 27, 1960)

$\mathbf{B}^{\mathrm{A}}$ ANDS belonging to the infrared spectrum of the diimide $\left(\mathrm{N}_{2} \mathrm{H}_{2}\right)$ molecule have been observed. This molecule has been previously identified by Foner and Hudson ${ }^{1}$ by mass spectrometry as a product of a radio-frequency discharge though gaseous hydrazine. We have used the method of preparation of Foner and Hudson. Our experimental arrangement permits deposition of the discharge products on a cold infraredtransparent window and simultaneous sampling of the

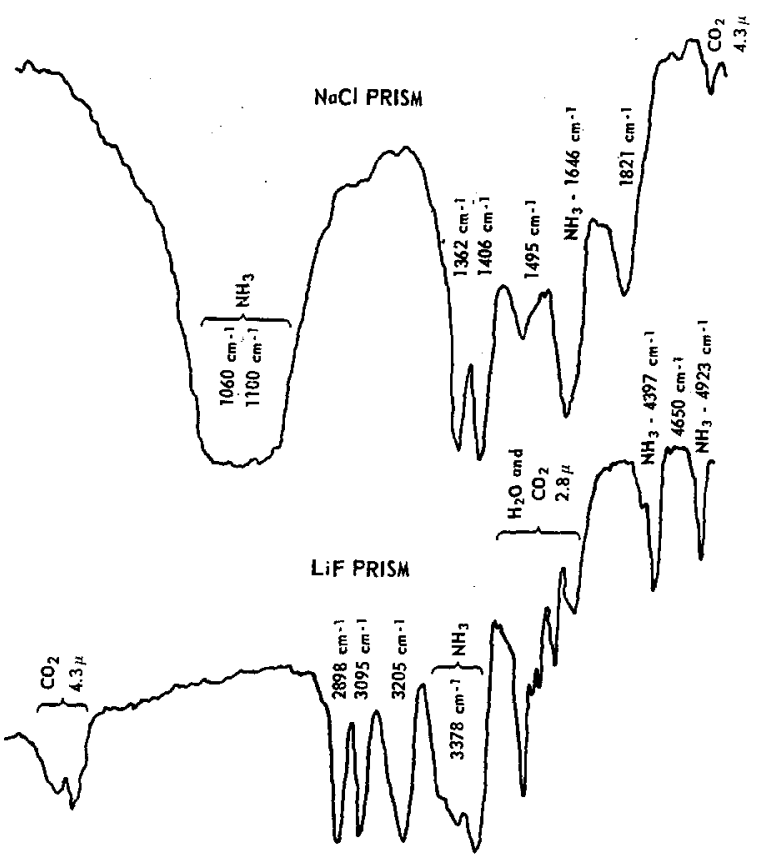

Frg. 1. Infrared spectrum of diimide.

discharge products for analysis on the mass spectrometer. A dry-ice trap immediately follows the discharge to remove hydrazine. The cryostat is very similar to one described by Wagner and Hornig. ${ }^{2}$ It has a clear aperture of $35 \mathrm{~mm}$ and fits easily into the sample space of the modified Perkin-Elmer model 12B spectrometer used in these experiments. With liquid nitrogen as coolant, a calcium fluoride window reached a steady temperature of $82^{\circ} \mathrm{K}$ in $15 \mathrm{~min}$ and a sodium chloride window $86^{\circ} \mathrm{K}$ in a similar time.

If hydrazine vapor is admitted into the evacuated system, it condenses completely in the dry-ice trap and nothing deposits on the cold window of the cryostat. When the discharge is started, the pressure following the dry-ice trap increases to between 100 and $1000 \mu$, depending on the discharge power. A yellow deposit, which is stable at least 2 to $3 \mathrm{hr}$ at temperatures below $120^{\circ} \mathrm{K}$, forms on the cold window.

Analysis of the discharge products with the mass spectrometer shows the presence of nitrogen, ammonia, and diimide. The undecomposed hydrazine is completely collected in the dry-ice trap as indicated by its absence in the mass spectrometer. In some experiments a trace of mass $45\left(\mathrm{~N}_{3} \mathrm{H}_{3}\right)$ was detected. By varying the hydrazine flow rate and the discharge power, it was possible to vary the ratio of diimide to ammonia from below $1 \%$ to about $20 \%$.

The spectrum of the deposit as recorded with the $\mathrm{NaCl}$ and LiF prisms is shown in Fig. 1. The rock salt region was examined as far as $15 \mu$, but no bands were found beyond those shown in the tracing. In addition to the bands of solid ammonia, ${ }^{3}$ the spectrum includes 